\title{
Epididymo-Orchitis and Testicular Abscess Caused by Salmonella enteritidis in Immunocompromised Patients in Kuwait
}

\author{
Khalid Al-Obeid ${ }^{a}$ Nawaf N. Al Khalifan ${ }^{b}$ Wafaa Jamal $^{a}$ Elijah O. Kehinde $^{\text {b, c }}$ \\ Vincent O. Rotimi ${ }^{a, c}$ \\ Departments of a Microbiology and ${ }^{b}$ Surgery (Division of Urology), Mubarak Al Kabeer Teaching Hospital and \\ ${ }^{\mathrm{c}}$ Faculty of Medicine, Kuwait University, Kuwait
}

\section{Key Words}

Epididymo-orchitis · Testicular abscess - Salmonella spp. $\cdot$ Immunocompromised patient $\cdot$ Kuwait

\begin{abstract}
Objective: To report relatively uncommon presentations of epididymo-orchitis and testicular abscess caused by Salmonella spp. in 2 immunocompromised patients. Clinical Presentation and Intervention: A 56-year-old man, a known case of systemic lupus erythematosus on azathioprine and prednisolone therapy, developed urinary tract infection followed by bacteremia and epididymo-orchitis. Both urine and blood cultures yielded Salmonella enteritidis strains, which were demonstrated by pulsed-field gel electrophoresis typing method to be genotypically identical. The second patient, a 55-year-old diabetic (type II), presented with a testicular abscess from which a pure culture of $S$. enteritidis was obtained. Both were treated with intravenous piperacillin and amikacin followed by oral ciprofloxacin, responded well to the therapeutic regimen and were discharged home well. Their follow-ups were uneventful. Conclusion: This report shows that it is important to consider Salmonella infection in the differential diagnosis of inflamed and tender testis in immunocompromised patients and to include blood, urine and stool cultures in all cases.
\end{abstract}

Copyright (c) 2006 S. Karger AG, Basel

\section{Introduction}

The commonest manifestation of salmonellosis is gastro-enteritis. Focal extra-intestinal infections are not as common and they usually follow complications with bacteraemia. An enlarged testis can be due to infectious and non-infectious diseases and until a diagnosis is made can evoke a great deal of anxiety in the patient as a prominent differential diagnosis is malignancy. Epididymo-orchitis complicating Salmonella infection in an immunocompetent or, even, immunocompromised patient is a rare condition. In a review of more than 700 cases of extra-intestinal infections caused by Salmonella spp., Cohen et al. [1] found only 12 cases (1.4\%) of orchitis or epididymitis. Since then only a handful cases of epididymo-orchitis and testicular abscesses associated with various strains of $\mathrm{Sal}$ monella spp., particularly in newborn babies and infants, have been reported [2-8]. There have been almost no cases of Salmonella epididymo-orchitis or testicular abscesses in adults.

In this communication, we present 2 cases of unusual presentation of Salmonella infection, epididymo-orchitis and testicular abscess in immunocompromised patients with systemic lupus erythematosus (SLE) and diabetes.

\begin{tabular}{ll}
\hline KARGER & ( ) 2006 S. Karger AG, Basel \\
Fax +4161306 1234 $341-7571 / 06 / 0154-0305 \$ 23.50 / 0$ \\
$\begin{array}{l}\text { E-Mail karger@karger.ch } \\
\text { www.karger.com }\end{array}$ & $\begin{array}{l}\text { Accessible online at: } \\
\text { www.karger.com } / \mathrm{mpp}\end{array}$
\end{tabular}




\section{Case Reports}

Case 1

A 56-year-old Jordanian man, who was a known case of SLE, on azathioprine and prednisolone, presented at our Emergency Room with 4-day history of progressive left testicular swelling, associated with localised pain, fever and rigors. He also complained of mild dysuria. The SLE (WHO class II) was diagnosed 5 years ago. In addition, he had a remarkable past medical history which included: hypertension (ranging between 180/100-190/110 mm $\mathrm{Hg}$ ), hypertrophic cardiomyopathy, hyperlipidaemia, hyperuricaemia, bilateral kidney stones, benign prostatic hyperplasia and druginduced pancytopenia. Ten days prior to this visit, he had suffered from a bout of urinary tract infection. Culture of his midstream urine yielded Salmonella enteritidis, sensitive to ampicillin, cefotaxime, chloramphenicol, piperacillin, piperacillin-tazobactam and ciprofloxacin, but resistant to amikacin, cephalothin and cefuroxime.

On examination, he looked toxic and was febrile (oral temperature $39.8^{\circ} \mathrm{C}$ ). His blood pressure was $180 / 110 \mathrm{~mm} \mathrm{Hg}$ and his left testicle was enlarged, hot, tender and erythematous. Other systems were normal. He was immediately admitted into the surgical ward for investigations and treatment. While in the ward, 3 sets of blood samples were taken for culture, haematological and biochemical investigations, and Brucella agglutination test. On day 2 of admission, urine and stool samples were taken for culture as well. WBC, $\mathrm{Hb}$ and platelet count were $6.4 \times 10^{9} / 1,9.9 \mathrm{~g} / \mathrm{dl}$ and $137 \times 10^{9} / 1$, respectively. Biochemical profile revealed slightly elevated urea $(10.6 \mathrm{mmol} / \mathrm{l})$; creatinine and other parameters were all within the normal ranges. Ultrasonography of the abdomen, pelvis, and scrotum showed mild hepatosplenomegaly, normal gallbladder, prostatic volume of $44 \mathrm{ml}$, normal-size right and left testes, thickened epididymis with decreased echogenicity and increased blood flow on Doppler of the left testicle. On suspicion of epididymo-orchitis, he was started initially on intravenous piperacillin, $2 \mathrm{~g} 8$-hourly and amikacin, $500 \mathrm{mg}$ 12-hourly. On day 4, results of urine and blood cultures revealed the presence of $S$. enteritidis resistant to amikacin. Consequently, amikacin therapy was discontinued. He improved within 5 days and was discharged home on oral ciprofloxacin, $500 \mathrm{mg}$ 12-hourly for 14 days. He has remained well 6 months after discharge with no recurrence of epididymo-orchitis.

Case 2

A 55-year-old diabetic (type II) Pakistani man, on oral hypoglycaemic agent for the previous 4 years, was admitted on September 29, 2004 through the Emergency Room because of a 4-day history of painful and swollen left scrotum. It was associated with fever of gradual onset. There was no diarrhoea, night sweats or fatigue. On examination, he was found to be in pain, anxious and febrile (oral temperature, $38^{\circ} \mathrm{C}$ ). His blood pressure was slightly raised $(140 / 90 \mathrm{~mm} \mathrm{Hg})$. All other systems were normal. The left scrotum was swollen, red, hot and tender. Ultrasound of the testes showed increased echo-pattern of the left testis surrounded by free fluid in its upper surface. A solid enhancement mass measuring $2.6 \times$ $2.5 \mathrm{~cm}$ was seen in the upper pole of the left testis in the region of the epididymis, which was suggestive of an abscess. A diagnosis of epididymitis and testicular abscess was made. Blood was taken for haematological and biochemical investigations as well as for culture as well. Urine and stool were also sent for microscopy and culture. On day 2 of admission, incision and drainage of the abscess was done and a specimen of pus was sent to the microbiology laboratory for culture. The results of the initial haematological and biochemical investigations were as follows: WBC $13.1 \times 10^{9} / 1$ (predominantly polymorphonuclear leucocytes); $\mathrm{Hb} 11.5 \mathrm{~g} / \mathrm{dl}$, and platelets $347 \times 10^{9} / 1$. Serum electrolytes, liver and renal function tests were normal. Serum glucose was $15.2 \mathrm{mmol} / \mathrm{l}$. Brucella agglutination test and VDRL test were negative. Urine microscopy showed $\mathrm{WBC}>200 / \mathrm{ml}$ and RBC $10 / \mathrm{ml}$, but the culture yielded no bacteria. Blood culture was negative. On day 4, culture of the pus yielded growth of $S$. enteritidis, sensitive to ampicillin, amoxicillinclavulanic acid, ceftriaxone, chloramphenicol, cotrimoxazole, ciprofloxacin and tetracycline and but resistant to amikacin, cephalothin, cefuroxime and gentamicin. Initial management was intravenous amikacin $500 \mathrm{mg}$ 12-hourly and ceftriaxone $2 \mathrm{~g}$ once daily. Upon receipt of results of culture and sensitivity, this was then changed to ceftriaxone $1 \mathrm{~g}$ once daily. He improved clinically and made uneventful recovery. He was discharged 7 days after admission on oral ciprofloxacin $500 \mathrm{mg}$ 12-hourly for 2 weeks. At followup 26 days later at the Urology OPD, he was well although the wound was still oozing. Culture of the wound and stool did not yield growth of Salmonella spp. He remains well 6 months after completing the above courses of antibiotics.

\section{Microbiological Investigations and Results}

In the Microbiology Laboratory, blood culture bottles were incubated inside the BACTEC 9240 blood culture machine (Becton, Dickinson \& Co., Sparks, Md., USA) and the midstream urine sample was inoculated onto cystine-lactose-electrolyte-deficient agar (Becton, Dickinson \& Co.) and blood agar (Tryptone Soya Agar, Oxoid Ltd., Basingstoke, Hampshire, England) supplemented with $5 \%$ sheep blood and incubated in air at $37^{\circ} \mathrm{C}$ for $24 \mathrm{~h}$. The pus sample was inoculated onto a set of selective and non-selective media and incubated aerobically and anaerobically. Both blood and urine cultures from case 1 yielded growth of Gram-negative bacilli which were later identified as $S$. enteritidis by the Vitek Aerobic Identification System (ID-GNI; REF 21312) (BioMérieux Inc., Marcy-I'Etoile, France) and manual serogrouping with commercial antisera (Wellcome Diagnostics, Dartford, UK). The stool culture was negative. Only the pus obtained from case 2 also yielded $S$. enteritidis.

Susceptibility testing was initially done in the Vitek machine using AST-N020 REF 22029, and the results obtained tallied with those obtained by determining the minimum inhibitory concentrations using the Etest strips (AB Biodisk, Sweden). The three isolates were susceptible to ampicillin, ciprofloxacin, cefotaxime, ceftriaxone, chloramphenicol, piperacillin, and piperacillin-tazobactam, but resistant to amikacin, cephalothin and cefuroxime. Brucella agglutination test was negative.

To determine if both isolates obtained from case 1 were indeed related strains, DNA extracts of the blood and urine isolates were subjected to pulse-field gel electrophoresis (PFGE) typing. Briefly, the organisms were grown overnight in tryptic soy broth and then used to prepare agarose blocks using the lysis solution containing lysozyme $(1 \mathrm{mg} / \mathrm{ml})$ instead of lysostaphin. Blocks were digested with $X b a \mathrm{I}$ (Gibco BRL) according to manufacturer's instruction. Electrophoresis was performed with $1 \%$ agarose gel in $\times 0.5 \mathrm{TBE}$ buffer using a CHEF DR III apparatus (Bio-Rad Laboratories). The gel was run at $14^{\circ} \mathrm{C}, 6 \mathrm{~V} / \mathrm{cm}$ and $120^{\circ}$ switch angle for $22 \mathrm{~h}$. Linear ramp of switch times was $0.5-40 \mathrm{~s}$. The gels were stained in $0.5 \mathrm{mg} / \mathrm{l}$ ethidium bromide and photographed under UV illumina- 
tion. The chromosomal patterns were examined and compared visually and then assigned to PFGE types. The strains in lanes 2 and 3 have identical DNA bands, indicating that they were identical strains; lane 1 contained the control DNA bands (fig. 1).

\section{Discussion}

Salmonella infections can present in many ways. Prominent amongst these is the gastro-enteritis, which occurs in about $68 \%$ of cases, followed by enteric fever $(8.8 \%)$, often presenting as 'pyrexia of unknown origin', focal manifestations (7.4\%) and asymptomatic carriers $(15.8 \%)$ [9]. Involvement of the genito-urinary tract is unusual and is often associated with congenital abnormalities, malignancy and immunosuppression. In one of the largest, and often quoted, series of cases ever reported on Salmonella infections from New York Salmonella Center, Saphra and Winter [9] indicated that of the 7,779 cases studied, only $49(0.6 \%)$ involved the genitourinary tract, mainly pyelonephritis. It is noteworthy that there was no single case of epididymitis or orchitis in this large series. Since then only a few sporadic cases of epididymitis and/or orchitis caused by Salmonella spp. have been reported, mainly in infants [2, 5, 6, 8]. These illustrate the rarity of the infection in adults.

Epididymo-orchitis is an inflammatory reaction which may be due to infectious or non-infectious aetiology. Bacterial aetiological cause is always a possibility but the least expected is Salmonella spp., especially in adult patients with no known compelling predisposing factor such as sickle cell disease. However, SLE, which was the co-morbidity in the first case, corticosteroid therapy and lymphoma are also major predisposing factors for invasive extra-intestinal Salmonella infections, where it presents mainly as bacteremia [10]. Patient 1 with SLE was on double immunosuppressive therapy including steroids and azathioprine. Urinary tract infection caused by Salmonella spp. is not totally unexpected in this patient but epididymo-orchitis has hardly ever been described as a complication of this infection in SLE patients and there is no record of such an infection reported in the Middle East literature.

The most probable route of infection in infective epididymo-orchitis or testicular abscess is the haematogenous route $[2,3]$ originating from the bladder as in the first case. This is hardly surprising as spontaneous septicaemia in adult males usually has as its main source urinary tract infections. Positive blood and urine cultures in our first patient support this assertion. However, bacter-

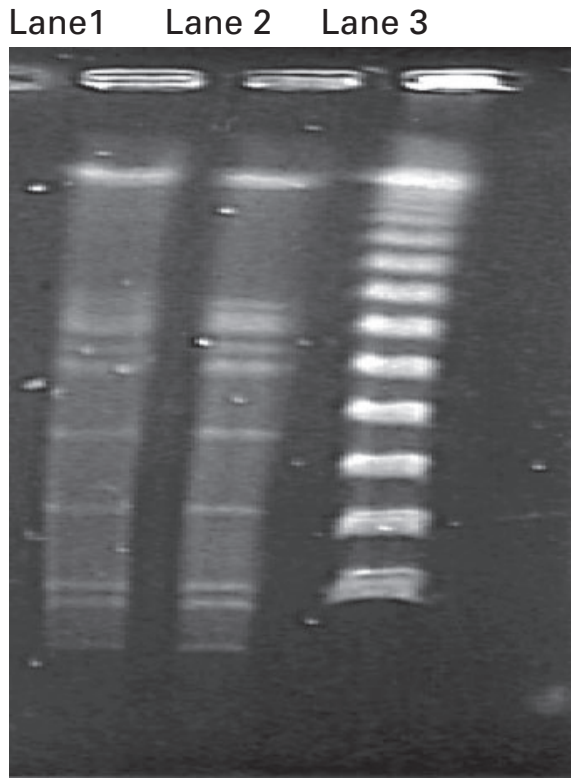

Fig. 1. PFGE of $X b a \mathrm{I}$-digested chromosomal DNA of two strains of $S$. enteritidis. Lane $1=S$. enteritidis from the blood; lane $2=$ $S$. enteritidis from urine; lane $3=$ standard $\mathrm{kb}$ marker.

aemia may be intermittent and the culture may be negative as in the second patient. Ascending infection is another route of infection via the urethra and vas deferens as well as outflow obstruction due to benign prostate hypertrophy. All these conditions were present in the SLE patient. The bacterial strains recovered from the blood and urine culture were phenotypically and genotypically identical, an indication that they were from the same source, most probably the gut. Although the culture of the stool specimens from both patients did not yield Salmonella spp., that the gut is the most probable source of both infections is high on the list of routes of infection. It is believed that immunosuppression in these patients was a major predisposing factor.

Our second case was a diabetic patient whose condition was poorly controlled and comes from an Asian country where Salmonella infection is endemic. Although Salmonella infection is a widespread zoonotic infection in developing countries, complication with epididymoorchitis is very rare. Diabetes mellitus as a predisposing factor is even rarer. As far as we could find in the literature this is the second reported case of this infection in a diabetic patient. The first, and only, case until now was a 56-year-old insulin-dependent diabetic patient with recurrent Salmonella septicaemia and previous history of diarrhoea [11]. 
A summary of reports on genito-urinary tract Salmonella infections gathered from the literature shows that $S$. enteritidis is the commonest species that causes focal lesion of the male genital tract infection [4-6]. Our finding of $S$. enteritidis in our patients is concordant with these reports.

As far as we are aware these are the first case reports of Salmonella epididymo-orchitis and testicular abscess in SLE and diabetic patients, in particular in Kuwait and in the Middle East as a whole. This rarity of epididymoorchitis caused by Salmonella in Kuwait is also supported by our own personal experience. For instance, of the 235 patients with epididymo-orchitis treated in the Urology Unit of Mubarak Hospital from January 1999 till November 2004, this is the first case caused by Salmonella [Kehinde, unpublished observation]. In our experience, the few cases of infective epididymo-orchitis seen in Kuwait have been due Brucella spp. [12].

The management of this condition in the diabetic patient required surgical drainage as well as intravenous antibiotic therapy. About three decades ago, cases of Salmonella orchitis and/or epididymitis were eventually managed by orchiectomy to effect bacteriological cure. This was a needless line of management as the conditions are amenable to conservative antibiotic therapy. Our patients responded well to the antibiotic regimens and were discharged home well. The more recent line of management involves surgical exploration, which is performed in the majority of cases because of the strong suspicion of incarcerated hernia rather than infection, especially in children. The first patient improved dramatically on antibiotic therapy alone while the second one required surgical drainage of an abscess and antibiotic therapy. Although antibiotics are not recommended for treating $\mathrm{Sal}$ monella gastro-enteritis, antibiotic therapy is needed for extra-intestinal infections.

\section{Conclusion}

These cases show that it is important to consider Salmonella infection in the differential diagnosis of inflamed and tender testis in immunocompromised patients and to include blood, urine and stool cultures in all cases.

\section{References}

-1 Cohen JI, Bartlett JA, Corey GR: Extra-intestinal manifestations of salmonella infections. Medicine (Baltimore) 1987;66:349-388.

$>2$ Handrick W, Bergmann L, Spencker FB, Benneck J: Orchitis und epididymitis caused by Salmonella cholerae suis in a premature infant. Int J Urol Nephrol 1987;80:89-91.

$>3$ Kostalas G, Thomas E: Orchitis and testicular abscess formation as complications of Salmonella virchow gastroenteritis. Med J Aust 1973; 2:769-771.

4 Svenungsson B: Suppurative epididymo-orchitis due to Salmonella enteritidis. J Infect 1984; 8:64-66.
Berner R, Schumacher RF, Zimmerhackl LB, Frankenschmidt A, Brandis M: Salmonella enteritidis orchitis in a 10 week old boy. Acta Paediatr 1994;83:992-993.

-6 Foster R, Weber TR, Kleiman M, Grosfeld JL: Salmonella enteritidis: testicular abscess in a newborn. J Urol 1983;130:790-791.

7 Ackley A, Pickens R: Salmonella virchow testicular abscess. J Urol 1978;122:267-268.

$>8$ Hakim A, Bradley H: Salmonella epididymoorchitis in infancy and childhood. Clin Pediatr 1992;31:120-122.

$>9$ Saphra I, Winter J: Clinical manifestations of salmonellosis in man, an evaluation of 7,779 human infections identified at the New York Salmonella Center. N Engl J Med 1957;256: 1128-1134.
10 Lim E, Koh W-H, Loh S-F, Lam M-S, Howe $\mathrm{H}-\mathrm{S}$ : Non-typhoidal salmonellosis in patients with systemic lupus erythematosus: a study of fifty patients and a review of the literature. Lupus 2001;10:87-92.

11 Gerridzen RG, Ooi TC, McKendry JBR: Salmonella epididymitis with recurrent septicemia in diabetic patient. Urology 1981;17:172174.

12 Jamal WY, Al Sweih NA, Rotimi VO: Brucella epididymo-orchitis in Mubarak Hospital: prevalence and case reports. Kuwait Med J 1996;(suppl): 262-265. 